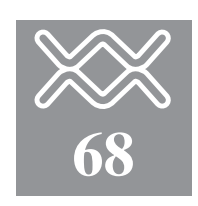

\title{
L'infezione da epatite C peggiora la prognosi dopo il trapianto di rene: esperienza di 400 trapiantati in un unico Centro
}

\author{
P. Stratta ${ }^{1}$, C. Musetti ${ }^{1}$ E. Turello ${ }^{1}$, E. Lazzarich ${ }^{1}$, D. Palmieri ${ }^{1}$, T. Cena ${ }^{2}$, C. Magnani ${ }^{2}$ \\ ${ }^{1}$ Cattedra di Nefrologia del Dipartimento di Medicina Clinica e Sperimentale \\ ${ }^{2}$ Cattera di Statistica dell'Università del Piemonte Orientale, Novara, Azienda Ospedaliera \\ Maggiore della Carità, Novara
}

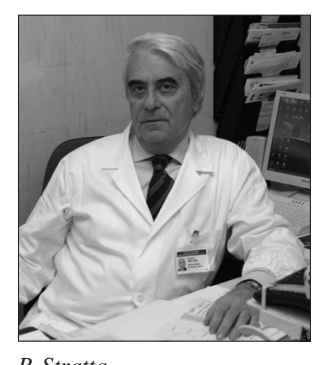

U infezione da HCV colpisce 150 milioni di persone nel mondo, causando un'epatite cronica nell' $85 \%$ dei casi, e cirrosi nel $15-30 \%$. È una infezione molto frequente tra i pazienti in dialisi e trapiantati di rene, con una prevalenza di circa il $20 \%$ nella popolazione trapiantata dell'Europa mediterranea.

Dal punto di vista dei rapporti tra $\mathrm{HCV}$ e rene, è ormai chiaro che il virus è in grado di causare un danno renale attraverso diversi meccanismi, per lo più mediati dalle conseguenze del tropismo linfocitario del virus stesso, tra cui la produzione di crioglobuline, potenzialmente responsabili di un peculiare tipo di coinvolgimento del parenchima del rene quale la glomerulonefrite membranoproliferativa. Dal punto di vista dei rapporti tra $\mathrm{HCV}$ e trapianto di rene, è oggi noto che i donatori $\mathrm{HCV}$ positivi (pari a 1$11 \%$ dei donatori totali), trasmettono l'infezione al ricevente, per cui è stata adottata in alcuni Centri la prassi di trapiantare il rene $\mathrm{HCV}$ positivo in riceventi HCV positivi.

La letteratura internazionale è concorde nel rilevare che la prognosi a lungo temine dei pazienti trapiantati di rene positivi per HCV è complessivamente peggiore rispetto ai pazienti $\mathrm{HCV}$ negativi, con una percentuale di morte più che raddoppiata al decimo anno (26\% vs $12 \%)$. Un'altra interessante osservazione è che anche le cause di morte dei pazienti trapiantati HCV positivi differiscono da quelle dei pazienti negativi, con una maggiore pre- valenza, nel gruppo dei pazienti positivi, di morti per cause infettive (45.5\% vs $22.9 \%$ ) e per insufficienza epatica (6\% vs $0.6 \%)$.

Anche il rischio di perdita dell'organo è aumentato nei pazienti trapiantati di rene $\mathrm{HCV}$ positivi rispetto ai pazienti HCV negativi, tanto che la sopravvivenza combinata rene-paziente è già ridotta dopo tre anni e mezzo di follow-up (86.9\% vs $91.5 \%)$ e si riduce ulteriormente durante la seconda decade dopo il trapianto (36\% vs $64 \%$ ). La patogenesi di questo danno non è del tutto chiarita, ma è stato comunque possibile rilevare alcuni elementi di rilievo potenzialmente responsabili di coinvolgimento renale, quali l'associazione con maggior proteinuria, maggiore incidenza di glomerulonefriti (membranosa e membranoproliferativa) e maggiore incidenza di crioglobulinemia. Inoltre, la presenza di crio- 


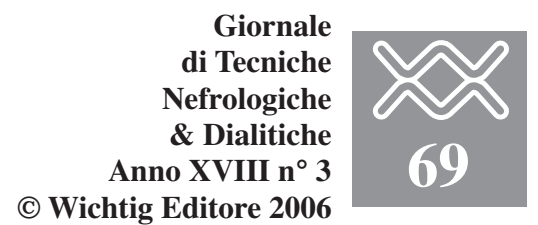

globulinemia nel paziente trapiantato HCV positivo risulta associata a una maggior morbilità, soprattutto di tipo neurologico e reumatologico. Infine, anche una maggiore incidenza di diabete mellito post-trapianto risulterebbe dimostrata nei pazienti HCV positivi, per quanto tale correlazione non sia molto forte.

Per quanto riguarda le scelte terapeutiche, in linea generale è condivisa l'osservazione che la terapia immunodepressiva debba essere particolarmente cauta nei pazienti trapiantati HCV positivi e quindi portatori di una infezione cronica associata a maggior rischio di mortalità e perdita del rene, con frequente e accurato controllo della viremia, così da poter avviare una precoce terapia specifica in caso di fasi di atti- vità della malattia. Tra i principali farmaci immunodepressori utilizzati nei trapianti, gli steroidi e gli inibitori delle calcineurine sono risultati associati a un aumento della replicazione virale in vivo, mentre i sieri policlonali antitimocitari e in minor misura anche gli antimetaboliti sono in grado di slatentizzare l'infezione inattiva.

La terapia antivirale con interferone ha ottenuto buoni risultati nei pazienti in dialisi, ma nel trapianto di rene i risultati sono ancora discussi, dal momento che il suo utilizzo è stato associato a un aumentato rischio di rigetto acuto e di perdita del rene. Inoltre, nonostante la temporanea riduzione del carico virale, alla sospensione dell'interferone si verifica un importante effetto "rebound". Per contro, la monoterapia con Ribavi- rina nel trapianto renale non è ancora supportata da studi prospettici, seppure sia stata utilizzata con successo in alcune esperienze isolate.

\section{L'esperienza del Centro Trapianti di Novara}

Uno studio sul ruolo della presenza di HCV positività nei pazienti trapiantati di rene è stata effettuata tra tutti i pazienti trapiantati presso il Centro Trapianti di Novara dal 4 novembre 1998 , data dell'esecuzione del primo trapianto, fino alla fine del 2005: si tratta di un totale di 400 pazienti, osservati per un follow-up medio di 34 mesi (minimo 3 mesi, massimo 83 mesi). I pazienti trapiantati $\mathrm{HCV}$ positivi sono risultati $52 / 400$, pari al $13 \%$ del to-

TABELLA I - CONFRONTO TRA LE SOTTOPOPOLAZIONI DI TRAPIANTATI RENALI HCV POSITIVI E NEGATIVI DEL CENTRO TRAPIANTI DI NOVARA. DESCRIZIONE DEMOGRAFICA, CLINICA, LABORATORISTICA E DATI ANALITICI DELL'ANALISI DI SOPRAVVIVENZA

\begin{tabular}{|c|c|c|c|c|}
\hline Variabile & $\begin{array}{l}\text { HCV negativi } \\
\quad n=348\end{array}$ & $\begin{array}{c}\text { HCV positivi } \\
\quad n=52\end{array}$ & $\begin{array}{c}\text { Totale } \\
n=400\end{array}$ & $\mathbf{p}$ \\
\hline Uomini & $204 / 348$ & $53.8 \%$ & $232 / 400$ & 0.54 \\
\hline Donne & $144 / 348 \quad 41.4 \%$ & $24 / 52 \quad 46.2 \%$ & $168 / 400 \quad 42.3 \%$ & \\
\hline Primo trapianto & $317 / 348 \quad 91.1 \%$ & $31 / 52 \quad 59.6 \%$ & $348 / 400 \quad 77 \%$ & 0.0001 \\
\hline Età al trapianto & $49 \pm 13$ & $46 \pm 9$ & $48 \pm 12$ & 0.0324 \\
\hline Mismatch totali & $2.72 \pm 0.96$ & $2.44 \pm 1.05$ & $2.69 \pm 0.98$ & 0.073 \\
\hline Età del donatore & $48 \pm 17$ & $49 \pm 13$ & $48 \pm 17$ & 0.466 \\
\hline Picco PRA & $11 \pm 21$ & $27 \pm 33$ & $13 \pm 23$ & 0.0009 \\
\hline Tempo ischemia fredda & $19 \pm 5.65$ & $20.7 \pm 5.06$ & $19.2 \pm 5.60$ & 0.03 \\
\hline BMI & $23.4 \pm 3.24$ & $23.3 \pm 3.80$ & $23.4 \pm 3.31$ & 0.888 \\
\hline Ritardo funzionale & $72 / 348 \quad 20.7 \%$ & $16 / 52 \quad 31 \%$ & $88 / 400 \quad 22 \%$ & 0.14 \\
\hline Rigetti & $38 / 348 \quad 10.9 \%$ & $11.5 \%$ & $44 / 400$ & 0.96 \\
\hline Diabete post. trapianto & $25 / 348 \quad 7.2 \%$ & $4 / 52 \quad 7.7 \%$ & $29 / 400 \quad 7.3 \%$ & 0.99 \\
\hline Creatinina dimissioni & $2.00 \pm 1.22$ & $2.35 \pm 1.26$ & $2.04 \pm 1.22$ & 0.067 \\
\hline Creatinina a 6 mesi & $1.71 \pm 0.62$ & $1.78 \pm 0.55$ & $1.72 \pm 0.61$ & 0.458 \\
\hline Proteinuria dimissioni & $0.47 \pm 0.306$ & $0.47 \pm 0.27$ & $0.47 \pm 0.29$ & 0.915 \\
\hline Proteinuria a 6 mesi & $0.23 \pm 0.31$ & $0.42 \pm 0.65$ & $0.25 \pm 0.37$ & 0.085 \\
\hline Sopravvivenza paziente & & & & 0.91 \\
\hline 3 mesi & 98.5 & 98.0 & 98.5 & \\
\hline 12 mesi & 97.9 & 96.0 & 97.7 & \\
\hline 2 anni & 97.5 & 96.0 & 97.3 & \\
\hline 5 anni & 95.8 & 96.0 & 95.9 & \\
\hline Sopravvivenza rene & & & & 0.001 \\
\hline 3 mesi & 99.1 & 94.2 & 98.5 & \\
\hline 12 mesi & 98.5 & 89.9 & 97.4 & \\
\hline 2 anni & 98.1 & 89.9 & 97.0 & \\
\hline 5 anni & 97.1 & 83.4 & 95.3 & \\
\hline
\end{tabular}


tale della popolazione arruolata. Il confronto con i pazienti HCV negativi ha dimostrato una percentuale significativamente maggiore di pazienti al loro secondo o terzo trapianto $(40 \%$ vs $9 \%$ ), un'età al trapianto lievemente minore (46 \pm 9 vs $49 \pm 13$ anni) e una maggiore immunizzazione (picco di PRA) (Tab I). Il tasso di ritardo nella ripresa funzionale (intesa come necessità di dialisi dopo il trapianto) non mostra significative differenze, così come pure il tasso di rigetti e il diabete insorto de novo dopo il trapianto. Per contro, risultano più elevati i valori di creatininemia alle dimissioni $2.35 \pm 1.23 \mathrm{mg} / \mathrm{dL}$ vs $2.0 \pm 1.22 \mathrm{mg} / \mathrm{dL}$ ) così come la proteinuria al sesto mese $(0.42 \pm 0.65 \mathrm{~g} / 24$ ore vs $0.23 \pm 0.31$ $\mathrm{g} / \mathrm{dL}$ ) pur non risultando le differenze statisticamente significative (Tab. I). Lo studio della sopravvivenza del paziente tramite metodo di Kaplan-Meyer non mostra differenze significative tra le due popolazioni in analisi, verosimilmente in relazione al periodo di osservazione relativamente breve rispetto all'andamento lento ed evolutivo dell'infezione HCV. Risulta invece confermata la maggior suscettibilità, anche precoce, alle gravi infezioni nei pazienti HCV positivi rispetto ai negativi: i 2 pazienti HCV positivi deceduti sono morti entrambi per cause postinfettive, contro i soli 2 morti per cause infettive tra i 12 deceduti dei 348 pazienti $\mathrm{HCV}$ negativi. Le infezioni fatali rappresentano una complicanza nel $3.8 \%$ di tutti i pazienti HCV positivi contro lo $0.6 \%$ di tutti i pazienti HCV negativi.

Inoltre, in linea con i dati di letteratura, i risultati della sopravvivenza del rene sono nettamente peggiori nei pazienti HCV positivi (a 1 anno dell' $89.9 \%$ vs $98.5 \%$, a 5 anni di $83.4 \%$ vs $97.1 \%$ ) (Fig. 1), con un rischio relativo superiore a 3 volte, che si mantiene tale anche nella analisi multivariata di Cox comprensiva degli altri principali fattori di rischio noti in grado di aumentare la probabilità di perdita del rene, quali età al trapianto, malattie sistemiche, ritardo nella ripresa funzionale, numero di rigetti, livello di anticorpi $(\mathrm{HR}=3.47$, con intervallo di confidenza al 95\%: 1.30-9.24)

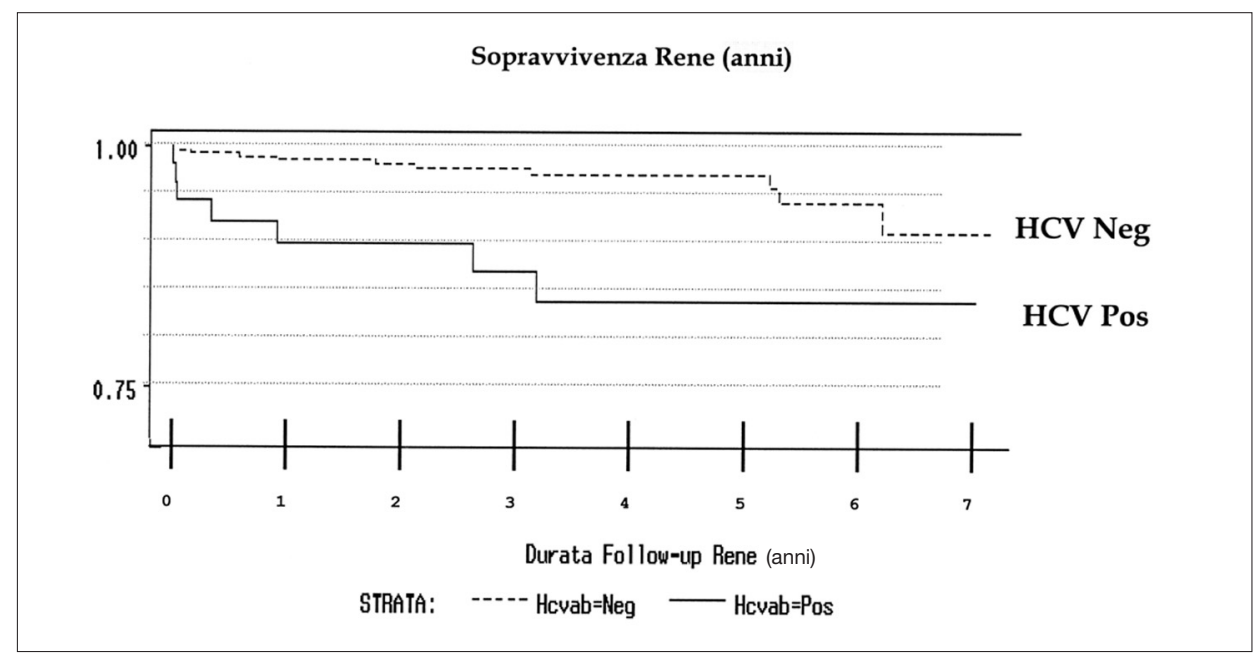

Fig. 1 - Analisi di Kaplan Meier della sopravvivenza del rene (endpoint=rientro in dialisi) dei pazienti HCV positivi e HCV negativi: si noti come la perdita del rene sia presente già nei primi tempi del follow-up.

\section{CONCLUSIONI}

Nella popolazione di 400 pazienti trapiantati presso il Centro Trapianti di Novara annesso alla Cattedra di Nefrologia dell'Università del Piemonte Orientale diretta dal professor Piero Stratta, l'analisi comparativa del gruppo di 52 pazienti HCV positivi ha consentito di rilevare come la sopravvivenza del paziente fino al quinto anno non sia ancora condizionata dalla positività $\mathrm{HCV}$, mentre il rischio di perdita del rene negli $\mathrm{HCV}$ positivi sia già significativamente aumentato, come evidenziato sia all'analisi univariata che multivariata di Cox ( $R R=3.47)$. Questo studio conferma come la popolazione in lista di attesa per trapianto renale $\mathrm{HCV}$ positiva costituisca un gruppo di pazienti complessivamente a prognosi più sfavorevole, sia dal punto di vista del rischio infettivo (in grado di condizionare, sul lungo termine, un aumentato rischio di morte), che della sopravvivenza dell'organo trapiantato.

Queste evidenze sottolineano come sia necessario, da un parte, identificare modalità di controllo e scelte terapeutiche modulate in funzione specifica di questa condizione, e, dall'altra, ridiscutere le indicazioni alla terapia antivirale specifica in fase di trattamento dialitico nel momento della immissione in lista di trapianto renale, quando la negativizzazione della viremia HCV potrebbe essere inserita tra gli obiettivi prioritari proprio in funzione delle ricadute di questa infezione sulla prognosi tardiva post-trapianto. 


\section{Giornale \\ di Tecniche \\ Nefrologiche \\ \& Dialitiche \\ Anno XVIII n ${ }^{\circ} 3$ \\ (C) Wichtig Editore 2006

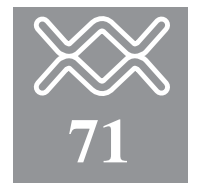

\section{BIBLIOGRAFIA}

1. Morales JM, Campistol JM, Dominguez-Gil B. Hepatitis C virus infection and kidney transplantation. Semin Nephrol 2002; 22: 365-74.

2. Manga Sahin G, Sahin S, Kantarci G, Ergin H. Impact of hepatitis $\mathrm{C}$ virus infection on patient and graft survival in kidney transplantation. Transplant Proc 2006; 2: 499-501.

3. Lin HH, Huang CC, Huang JY, Yang CW, Wu MS, Fang JT, Yu CC, Chiang YJ, Chu SH. Impact of HCV infection on first cadaveric renal transplantation, a single center experience. Clin Transplant 2004; 3: 261-6.

4. Sens YAS, Malafronte P, Souza JF, Bruno S, et al. Cryoglobulinemia in Kidney Transplant Recipients. Transpl Proc 2005; 37: 4273-5.

5. Abbott KC, Krista LL, Bucci JR, Agodoa LY, Peters TG, Schnitzler MA. The impact of transplantation with deceased donor hepatitis C-positive kidney on survival in wait-listed long-term dialysis patients. Am J Transpl 2004; 4: 2032-7.

6. Batty DS Jr, Swanson SJ, Kirk AD, Ko CW, Agodoa LY, Abbott KC. Hepatitis $C$ virus seropositivity at the time of renal transplantation in the United States: associated factors and patient survival. Am J Transplant 2001; 1: 179-84.

7. Baid S, Cosimi AB, Tolkoff-Rubin N, Colvin RB, Williams WW, Pascual M. Renal disease associated with hepatitis $\mathrm{C}$ infection after kidney and liver transplantation. Transplantation 2000; 70: 255-61.

8. Morales JM, Campistol JM. Transplantation in the patient with hepatitis C. J Am Soc Nephrol 2000; $11(7): 1343-53$.

9. Pereira BJ, Natov SN, Bouthot BA, et al. Effects of hepatits C infection and renal transplantation on survival in end-stage renal disease. The New England Organ Bank Hepatitis C Study Group. Kidney Int 1998; 53 : 1374-81.
10. European Best Practice Guidelines for Renal Transplantation (part 1). Nephrol Dial Transplant 2000; 15(Suppl 7): S1-85.

11. Hanafusa T, Ichikawa Y, Kishikawa $\mathrm{H}$, et al. Retrospective study on the impact of hepatitis $\mathrm{C}$ virus infection on kidney transplant patients over 20 years. Transplantation 1998; 66: 4, 471-6. 